\title{
Amorphous silica nanoparticles size-dependently aggravate atopic dermatitis-like skin lesions following an intradermal injection
}

Toshiro Hirai ${ }^{1,2}$, Tomoaki Yoshikawa ${ }^{1,2^{*}}$, Hiromi Nabeshi ${ }^{1,2}$, Tokuyuki Yoshida ${ }^{1,2}$, Saeko Tochigi ${ }^{1,2}, K_{0}$-ichi Ichihashi ${ }^{1,2}$, Miyuki Uji ${ }^{1,2}$, Takanori Akase ${ }^{1,2}$, Kazuya Nagano ${ }^{2}$, Yasuhiro Abe ${ }^{2}$, Haruhiko Kamada ${ }^{2,3}$, Norio Itoh ${ }^{1,2}$, Shin-ichi Tsunoda ${ }^{2,3}$, Yasuo Yoshioka ${ }^{2,3}$ and Yasuo Tsutsumi ${ }^{1,2,3^{*}}$

\begin{abstract}
Background: Due to the rising use of nanomaterials (NMs), there is concern that NMs induce undesirable biological effects because of their unique physicochemical properties. Recently, we reported that amorphous silica nanoparticles (nSPs), which are one of the most widely used NMs, can penetrate the skin barrier and induce various biological effects, including an immune-modulating effect. Thus, it should be clarified whether nSPs can be a risk factor for the aggravation of skin immune diseases. Thus, in this study, we investigated the relationship between the size of SPs and adjuvant activity using a model for atopic dermatitis.

Results: We investigated the effects of nSPs on the AD induced by intradermaly injected-mite antigen Dermatophagoides pteronyssinus (Dp) in NC/Nga mice. Ear thickness measurements and histopathological analysis revealed that a combined injection of amorphous silica particles (SPS) and Dp induced aggravation of AD in an SP size-dependent manner compared to that of Dp alone. In particular, aggravation was observed remarkably in nSPinjected groups. Furthermore, these effects were correlated with the excessive induction of total IgE and a stronger systemic Th2 response. We demonstrated that these results are associated with the induction of IL-18 and thymic stromal lymphopoietin (TSLP) in the skin lesions.

Conclusions: A particle size reduction in silica particles enhanced IL-18 and TSLP production, which leads to systemic Th2 response and aggravation of AD-like skin lesions as induced by Dp antigen treatment. We believe that appropriate regulation of nanoparticle physicochemical properties, including sizes, is a critical determinant for the design of safer forms of NMs.
\end{abstract}

Keywords: Nanoparticle, Silica, Allergy, Cytokines

\section{Background}

With the development of nanotechnology, practical uses for nanomaterials (NMs) are rapidly spreading to a wide variety of fields, such as cosmetics, food, and medicine, because they have unique physicochemical properties and exert innovative functions [1-3]. These observations mean that intentional exposure of NMs is unavoidable in everyday life. However, it is a concern that NMs can exhibit

\footnotetext{
* Correspondence: tomoaki@phs.osaka-u.ac.jp; ytsutsumi@phs.osaka-u.ac.jp 'Laboratory of Toxicology and Safety Science, Graduate School of Pharmaceutical Sciences, Osaka University, 1-6, Yamadaoka, Suita, Osaka 5650871, Japan

Full list of author information is available at the end of the article
}

unknown harmful effects $[4,5]$. For example, maternal exposure to titanium dioxide nanoparticles $\left(\mathrm{nTiO}_{2}\right)$ induces gene expression alterations related to brain development [6]. We also revealed that amorphous silica nanoparticles (nSPs) and $\mathrm{nTiO}_{2}$ induce reproductive and/or liver toxicity compared with submicron-sized amorphous silica particles (SPs) $[7,8]$. However, little information exists on the potential hazard of NMs. To ensure the safety of NMs and enjoy their many benefits, it is essential to obtain more information on the relationship between the factors associated with NM hazards and their physicochemical properties, such as size and surface properties, to design safer forms of NMs.

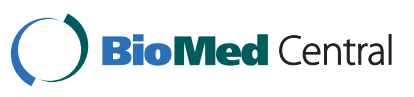

(c) 2012 Hirai et al; licensee BioMed Central Ltd. This is an Open Access article distributed under the terms of the Creative Commons Attribution License (http://creativecommons.org/licenses/by/2.0), which permits unrestricted use, distribution, and reproduction in any medium, provided the original work is properly cited. 
nSPs are used as a base material in cosmetics and an anti-caking agent in food because of their high transparency and coatability $[9,10]$. Because nSPs are one of the most widely used NMs, the chances of being exposed to nSPs in our daily life are high. Thus, we must analyze the in vitro/in vivo biodistribution of nSPs including estimations of whether they can penetrate the biological barrier. Additionally, we need to estimate whether they could be responsible for acute/ chronic side effects, thereby facilitating a more accurate risk analysis of nSPs. Under these circumstances, we revealed that $\mathrm{nSPs}$ with a diameter of $70 \mathrm{~nm}$ (nSP70) can penetrate the skin barrier and enter keratinocytes and Langerhans cells [11]. Our studies further revealed that a dermal application of nSP70 induces apoptosis in dermal cells [11]. In addition, we reported that nSPs have a greater cytotoxic effect on Langerhans cells [12]. Thus it is possible that nSPs may be associated with development of the allergic diseases such as atopic dermatitis (AD). However, the relationship between the size of SPs and its adjuvant activity which can affect aggravation of AD like skin lesion has remained unclear.

In present study, we investigated the effect of nSPs on AD-like skin lesions using Dermatophagoides pteronyssinus (Dp), which is the main allergen of AD [13], in $\mathrm{NC} /$ Nga mice. We also examined the relationship between the SP size and aggravation of AD.

\section{Results}

Physicochemical properties of variously sized SPs

Prior to undertaking this study, we first analyzed the physicochemical properties of SPs (Table 1). SPs remained as stable well-dispersed particles in PBS and not as aggregates. Thus, these particles were ideally suited to evaluate whether their biological effects depended on particle size.

\section{Effects of variously sized SPs on AD-like skin lesions}

To evaluate whether variously sized SPs affects AD-like skin lesions induced by $\mathrm{Dp}$, we first measured ear

Table 1 Summary of the physicochemical properties of amorphous silica particles (SPs).

\begin{tabular}{lllll}
\hline & $\begin{array}{l}\text { Primary particle } \\
\text { size }(\mathbf{n m})^{\mathbf{a}}\end{array}$ & $\begin{array}{l}\text { Diameter in } \\
\text { PBS }(\mathbf{n m})\end{array}$ & $\begin{array}{l}\text { Mean zeta } \\
\text { potential }(\mathbf{m V})\end{array}$ & pH \\
\hline mSP1000 & 1000 & $1136 \pm 32.1$ & $-33.2 \pm 1.4$ & 7.6 \\
nSP300 & 300 & $264 \pm 7.2$ & $-25.8 \pm 0.7$ & 7.4 \\
nSP100 & 100 & $106 \pm 0.6$ & $-24.3 \pm 0.5$ & 7.4 \\
nSP70 & 70 & $76 \pm 1.7$ & $-19.5 \pm 1.0$ & 7.4 \\
nSP30 & 30 & $39 \pm 4.2$ & $-14.0 \pm 1.3$ & 7.4 \\
\hline
\end{tabular}

Mean particle size and zeta potential in solution of silica particles are expressed as mean \pm S.D. $(n=3)$. ${ }^{\text {a }}$ Information from technical datasheet of products. thickness on days 0 and 19 . We confirmed that the intradermal injection of Dp enhanced ear thickening as compared to that of PBS (Figure 1A). A combined injection of Dp and the submicron-sized SPs, mSP1000 or nSP300, did not enhance ear thickening compared with that of Dp alone. In contrast, a combined injection of Dp + nSPs (nSP100, nSP70, and nSP30) enhanced ear thickening two- to fivefold compared with that of Dp alone. nSP-treatment showed a marked tendency to cause ear thickening. Thus, nSPs with a diameter $\leq 100$ $\mathrm{nm}$ acted specifically to enhance ear thickening in this model.

Next, we performed hematoxylin-eosin and toluidine blue staining using ear sections $24 \mathrm{~h}$ after the last intradermal injection (Figure $1 \mathrm{~B}$ and 1D). Then, several representative symptoms (scab, acanthosis, inflammatory cell infiltration, and scleredema) were scored on the $\mathrm{H} \& \mathrm{E}$ sections (Figure $1 \mathrm{C}$ ). A Dp injection caused significant inflammatory cell infiltration compared with a PBS injection. In contrast, a combined injection of Dp and variously sized SPs caused more severe inflammatory cell infiltration than that of Dp alone. Although the Dp injection did not cause scabs, acanthosis, or scleredema, the combined injection of Dp and SPs with a diameter $\leq 300 \mathrm{~nm}$ caused acanthosis. Furthermore, a combined injection of Dp and nSPs caused significant scabs and scleredema (Figure 1C). Mast cell infiltrates in the skin were stronger following the combined injection of $\mathrm{Dp}$ and nSPs (Figure 1D and 1E). Thus, the injection of Dp and submicron-sized SPs caused only inflammatory cell infiltration and acanthosis compared with that of Dp alone. However, injecting $\mathrm{Dp}$ and nSPs caused not only inflammatory cell infiltration and acanthosis but also scabs and scleredema. These findings suggest that injecting $\mathrm{nSPs}$ with a diameter $\leq 100 \mathrm{~nm}$ and Dp induced severe AD-like histological changes.

\section{Effects of variously sized SPs on the IgE response}

Many reports have shown that the total IgE and allergen-specific IgE level correlate with severity of AD $[14,15]$. To clarify the nSP-mediated aggravation of ADlike lesions, we first measured total IgE and Dp-specific IgE $24 \mathrm{~h}$ after the last intradermal injection (Figure 2). The total IgE in the Dp alone group was higher than that in the PBS group (Figure 2A). Furthermore, the levels of total IgE in the Dp + SP groups were enhanced in an SP size-dependent manner compared with those in the Dp alone group. A regression analysis showed that the particle size reduction was a significant contributor to this effect (Figure $2 \mathrm{~B} ; \mathrm{R}^{2}=0.98$ ). Therefore, it paralleled the severity of the AD-like skin lesions. In the presence of variously sized SPs, the Dp-specific IgE levels were significantly increased compared with Dp alone group (Figure 2C). However, Dp-specific IgE levels 
A

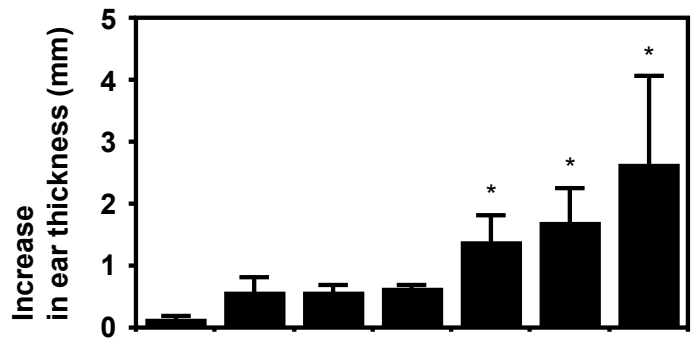

C
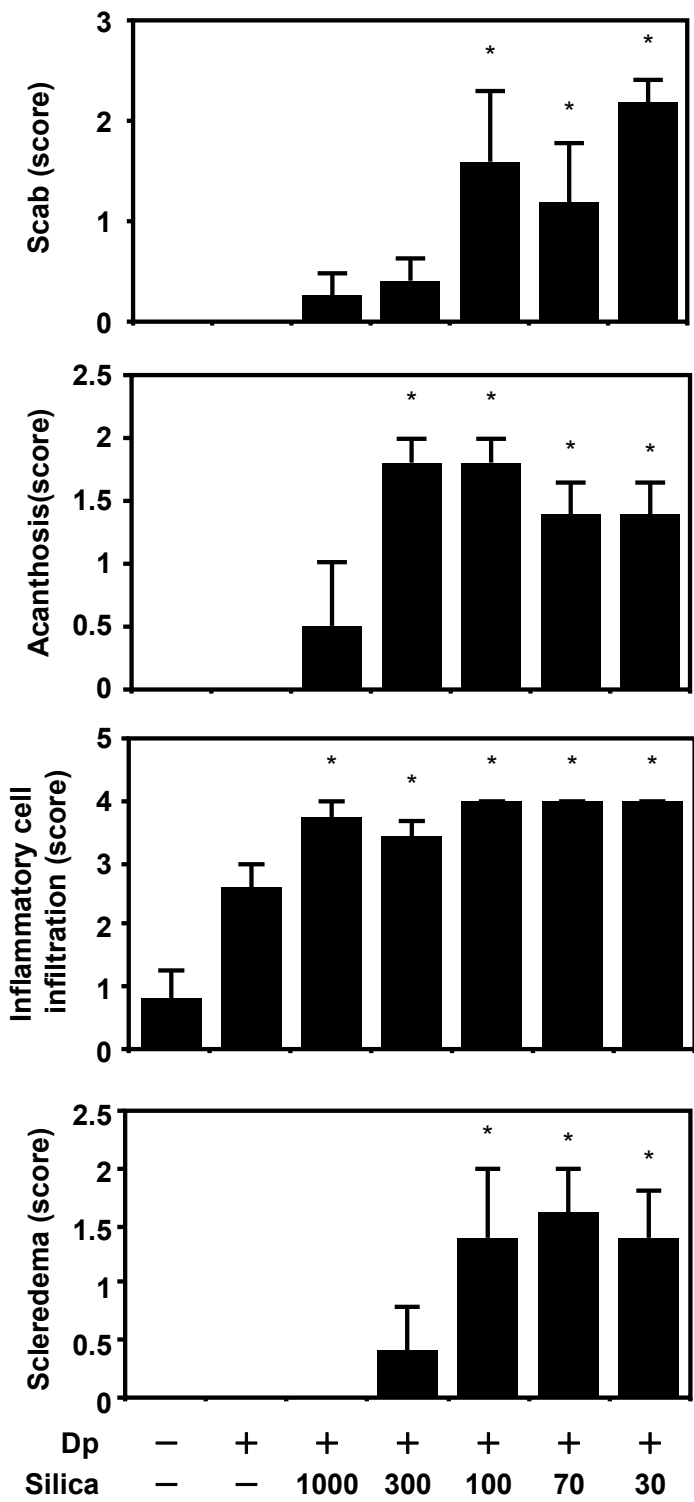

B

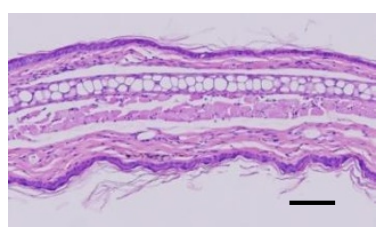

PBS

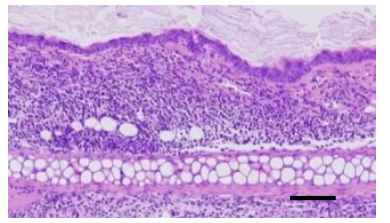

$D p+m S P 1000$

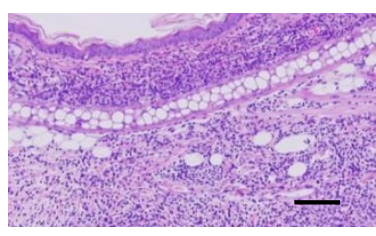

Dp

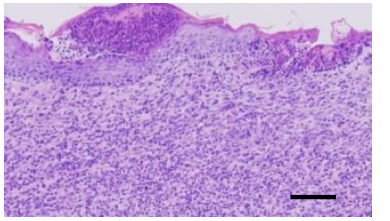

$D p+n S P 30$
D
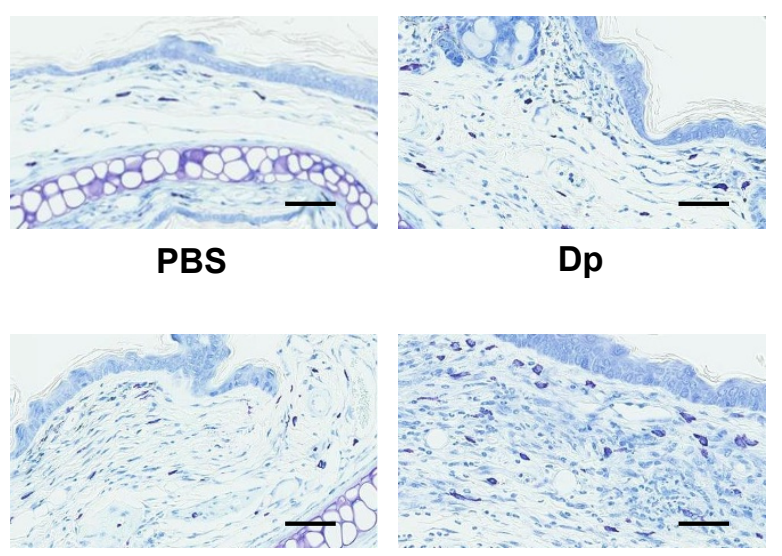

$D p+m S P 1000$

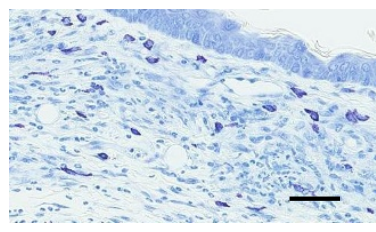

Dp + nSP30

E

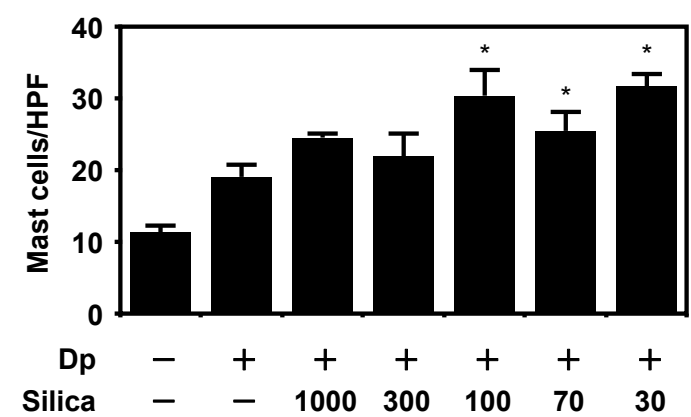

Figure 1 Effects of amorphous silica nanoparticles (nSPs) on atopic dermatitis (AD)-like skin lesions induced by Dermatophagoides pteronyssinus (Dp). NC/Nga mice were intradermally injected on the ventral side of both ears with Dp alone or Dp plus SPs in PBS on days 1 , $3,5,7,9,11,13,16$, and 18. Ear thickness was measured on days 0 and 19 (A). The ears were removed $24 \mathrm{~h}$ after the last intradermal injection. Sections were prepared, and the ears were stained with H\&E to assess representative symptoms of $A D(B$ and $C$ ) or with toluidine blue to assess mast cell infiltration ( $D$ and $E$ ). Representative histological findings of the PBS, Dp, Dp + mSP1000, and Dp + nSP30 groups are shown. Several representative symptoms of AD were scored in the H\&E sections (C). Infiltration of mast cells was evaluated as the number of cells in toluidine blue sections in a high power field (E). Data are presented as mean \pm SE of 4-5 animals per group. The results are representative of two separate experiments. ${ }^{*} \mathrm{P}<0.05$ vs. Dp group. Scale bars: $100 \mu \mathrm{m}(\mathrm{B})$ and $50 \mu \mathrm{m}$ (D). 


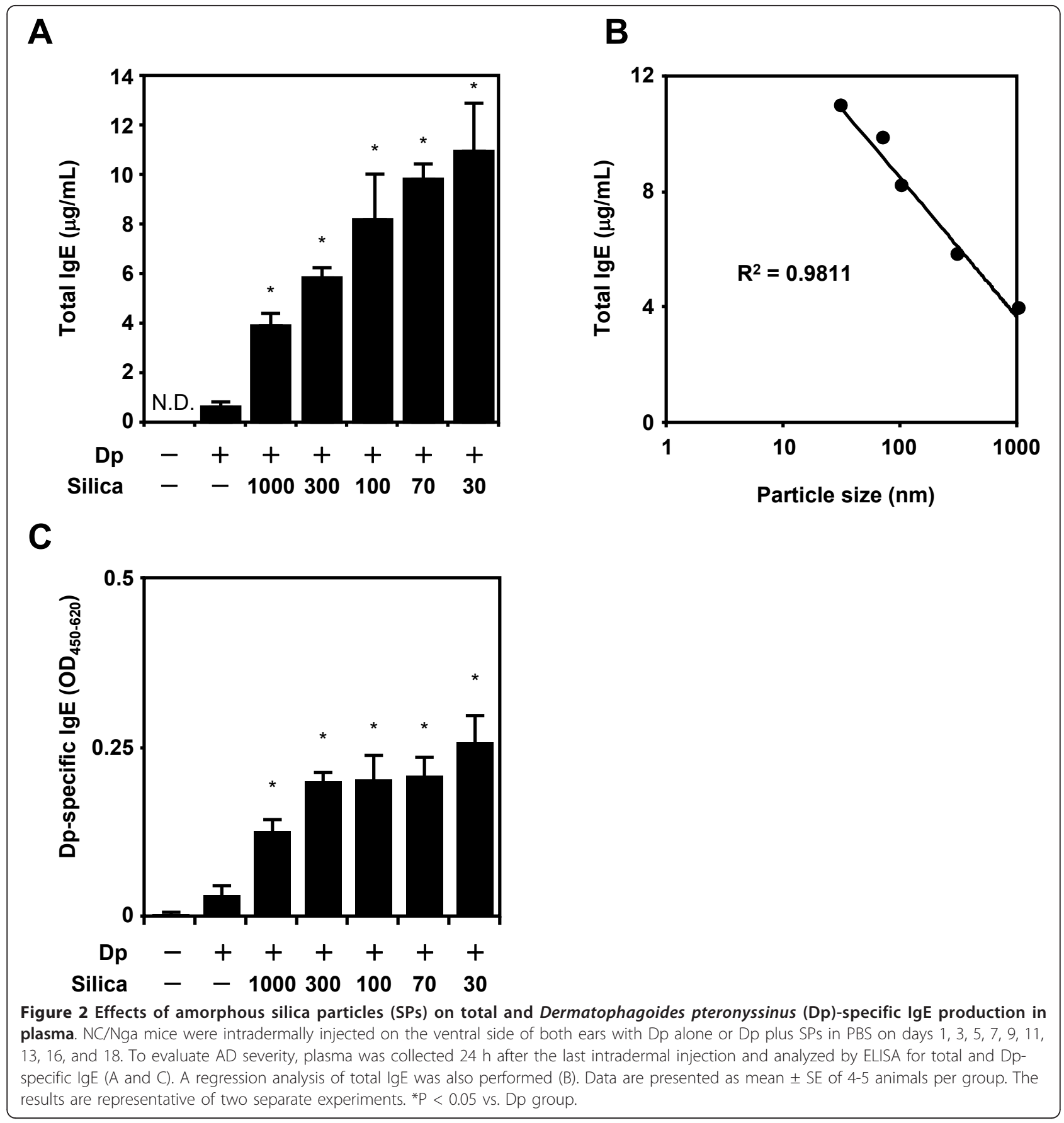

were not paralleled with the size of SPs. Thus, nSPmediated aggravation of AD-like lesions has more to do with total IgE than Dp-specific IgE.

\section{Cytokine expression analysis of nSP-injected skins}

To clarify the mechanism of AD-like skin lesions enhanced by SPs, we analyzed the cytokine expression profile in the skin related to $\mathrm{Dp}$. We examined the levels of Th2-type cytokines (IL-4, IL-5, and IL-13), Th1-type cytokines IFN- $\gamma$, IL-18, and thymic stromal lymphopoietin (TSLP) in the ears $24 \mathrm{~h}$ after the last intradermal injection (Figure 3). Intradermal injection of Dp alone increased the local expression of IL-4 and IL-13 and decreased the production of IL- 5 and IFN- $\gamma$ compared with PBS injection. Only differences of the expression of IL-13 between the Dp group and PBS group is significant. In contrast, the level of IL-4 in the Dp + SP groups with diameters $\leq 300 \mathrm{~nm}$ increased significantly 
A
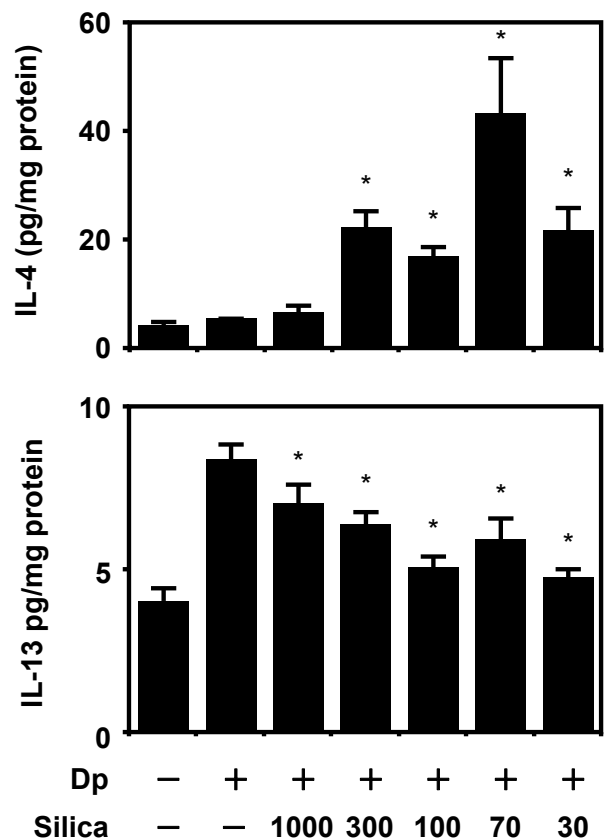

B

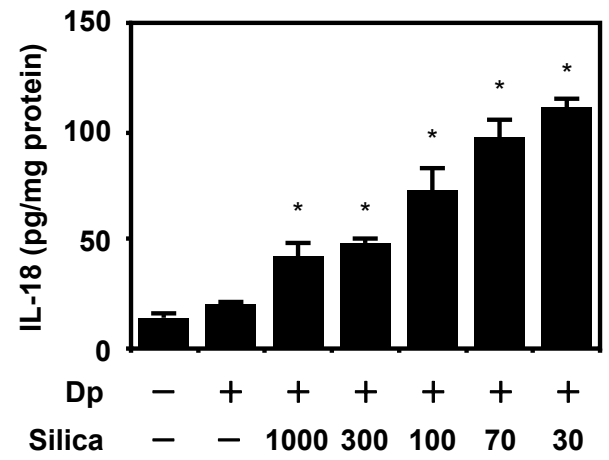

D

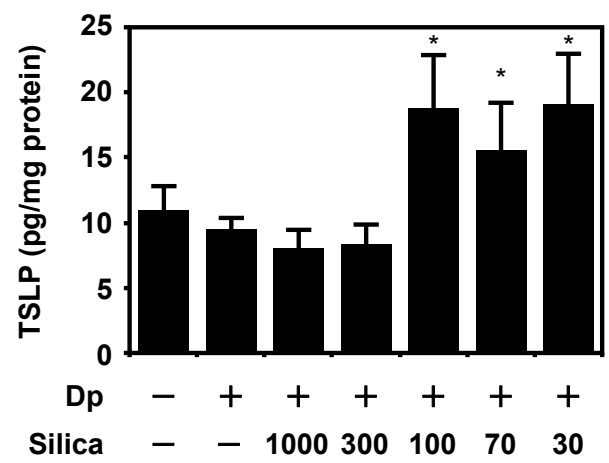

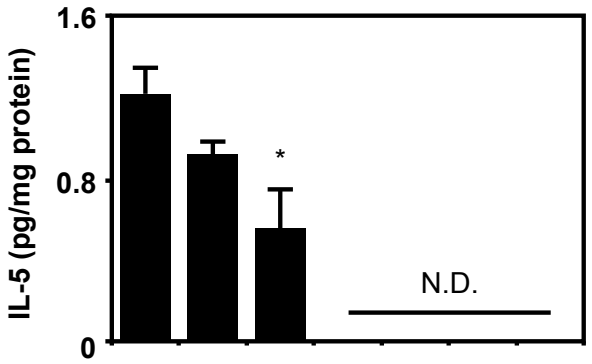

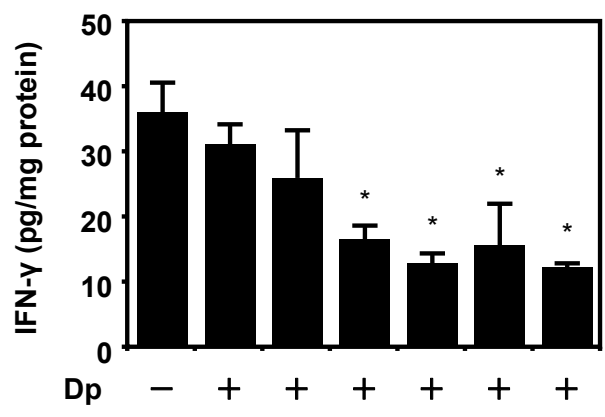

Silica --10003001007030

C

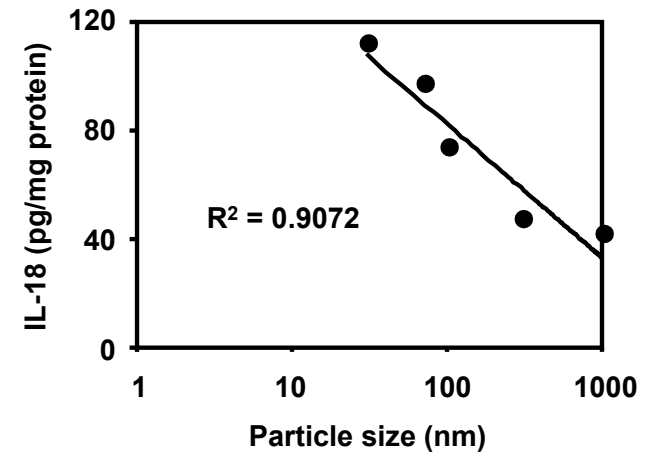

Figure 3 Analysis of the local immune response induced by Dermatophagoides pteronyssinus (Dp) + amorphous silica particles (SPs) NC/Nga mice were intradermally injected on the ventral side of both ears with Dp alone or Dp plus SPs in PBS on days 1, 3, 5, 7, 9, 11, 13, 16, and 18. At $24 \mathrm{~h}$ after the last intradermal injection, the ears were removed and homogenized. The homogenates were centrifuged at 10,000 rpm for $5 \mathrm{~min}$, and the supernatants were collected. The levels of interleukin (IL)-4, IL-5, IL-13, interferon (IFN)- $\gamma$, IL-18, and thymic stromal lymphopoietin (TSLP) in the supernatants were examined by ELISA (A, B, and D). A regression analysis of IL-18 was also performed (C). Data are presented as mean \pm SE of 4-5 animals per group. The results are representative of two separate experiments. ${ }^{*} P<0.05$ vs. Dp group. 
compared with that in the Dp alone group. The levels of IL-5 and IL-13 in the Dp + SP groups with a diameter $\leq 300 \mathrm{~nm}$ decreased significantly compared with those in the Dp alone group. Additionally, injections of SPs with a diameter $\leq 300 \mathrm{~nm}$ led to a significant decrease in IFN- $\gamma$ compared to those of Dp alone (Figure 3A). Thus, the injection of SPs with Dp tended to enhance the effects of injecting $\mathrm{Dp}$ alone in terms of cytokine levels in the skins. However, the levels of IFN-gammma and IL-5 were significantly reduced after SPs treatment when compared to Dp only. The levels of IL-18, known as an $\mathrm{AD}$-inducing cytokine, were higher in the $\mathrm{Dp}+$ smaller SP groups than those in the Dp alone group (Figure 3B). A regression analysis showed that the particle size reduction was a significant contributor to this effect (Figure $3 C ; R^{2}=0.91$ ). IL-18 production sizedependently increased similar to that of plasma total IgE levels. Furthermore, the levels of TSLP, an AD-inducing cytokine, were enhanced in only the $\mathrm{Dp}+\mathrm{nSP}$ groups compared with those in the $\mathrm{Dp}$ alone group (Figure $3 \mathrm{D})$. This result matched that of increase in ear thickness.

\section{Dp-specific immune response in Dp + SPs-injected mice}

To evaluate the effect of SPs on systemic immune responses, we examined the levels of Dp-specific IgG and its subtypes by ELISA $24 \mathrm{~h}$ after the last intradermal injection (Figure 4A and 4B). Higher levels of Dpspecific IgG were observed in the Dp + SP groups than those in the Dp alone group (Figure 4A). Furthermore, higher Dp-specific IgG levels were observed, particularly in Dp $+n S P$ groups. Dp-specific IgG1 levels in the Dp + SP groups were higher than those in the Dp alone groups. In contrast, the levels of Dp-specific IgG2a were low in all groups except the Dp + nSP30 group. Plasma IgG subclass responses have been used to assess the type of immune response. It is well-known that $\operatorname{IgG1}$ is indicative of a Th2-type response, whereas IgG2a is indicative of a Th1 response. Therefore, these results indicate that Dp + SPs induced antigen-specific Th2type immune responses and that Dp $+\mathrm{nSP} 30$ induced not only Th2-type immune responses but also Th1-type responses.

To further characterize the type of systemic immune response, we measured the levels of Dp-specific IFN- $\gamma$ and IL-4-secreting splenocytes from each intradermally injected mouse using a cytokine-specific ELISPOT assay (Figure 4B). The number of Dp-specific IFN- $\gamma$-secreting splenocytes in the Dp + nSP30 group was significantly greater than that in all other groups. However, no difference was observed in the Dp-specific IFN- $\gamma$-secreting splenocytes of all groups except the Dp + nSP30 group. These levels of Dp-specific IFN- $\gamma$-secreting splenocytes were correlated with Dp-specific IgG2a levels. The levels of Dp-specific IL-4-secreting splenocytes in the Dp + SP groups were enhanced in a SP size-dependent manner compared with those in the Dp alone group (Figure 4C). The regression analysis showed that the particle size reduction was a significant contributor to this effect (Figure 4D; $R^{2}=0.93$ ). Thus, the levels of Dp-specific IL-4-secreting splenocytes paralleled AD severity.

\section{Discussion}

The lifetime prevalence of AD is estimated at $15-30 \%$ in children and $2-10 \%$ in adults, and the incidence of $\mathrm{AD}$ has increased by two- to threefold during the past three decades in industrialized countries $[16,17]$. Although environmental factors and lifestyle changes are speculated to be associated with the increased number of patients with $\mathrm{AD}$, the detailed relationships between environmental factors or lifestyle changes and the increase in AD are not well understood. Some epidemiological studies have shown that chronic exposure to urban fine particles could be a risk factor for $\mathrm{AD}$ $[18,19]$. In addition, $\mathrm{nTiO}_{2}$ and polystyrene nanoparticles have been reported to exacerbate AD $[20,21]$. Thus, among other environmental factors, NMs may be a risk factor for the onset or aggravation of AD. However, the relationship between $\mathrm{AD}$ and $\mathrm{nSPs}$, which are one of the most widely used NMs in various applications, has never been investigated. Here, to clarify the relationship between NM use and the onset or aggravation of AD, we investigated the effects of nSPs on AD-like skin lesions induced by a mite allergen $\mathrm{Dp}$.

Typical symptoms of AD, such as inflammation or itching of the skin, are induced by chemical mediators such as histamine and some cytokines [22,23]. Furthermore, these chemical mediators or cytokines are induced by IgE or antigen cross-linking of IgE bound to FceRI on mast cells [24]. We measured Dp-specific IgE to determine allergen-specific IgE participation in the aggravation of $\mathrm{AD}$. As expected, the $\mathrm{Dp}$-specific IgE level was higher in the $\mathrm{Dp}+\mathrm{SP}$ groups than that in the Dp alone group (Figure 2C). Because particle materials influence the intracellular processing of antigen in antigen-presenting cells (APCs) such as dendritic cells (DCs) $[25,26]$, it is possible that SPs affected these APCs and changed the Dp-specific immune responses. However, these Dp-specific IgE levels were not dependent on SP size and were not high in the Dp + nSP groups compared with those in the Dp + submicron-sized SPs groups. In contrast, a regression analysis showed that the total IgE levels, which are known to correlate with AD severity $[27,28]$, were completely dependent on injected SP size (Figure 2A; $R^{2}=0.98$ ). Thus, this result suggested that smaller SPs induced more severe AD and, most importantly, that the mechanisms of $A D$ aggravation caused by SPs were induced not only by 


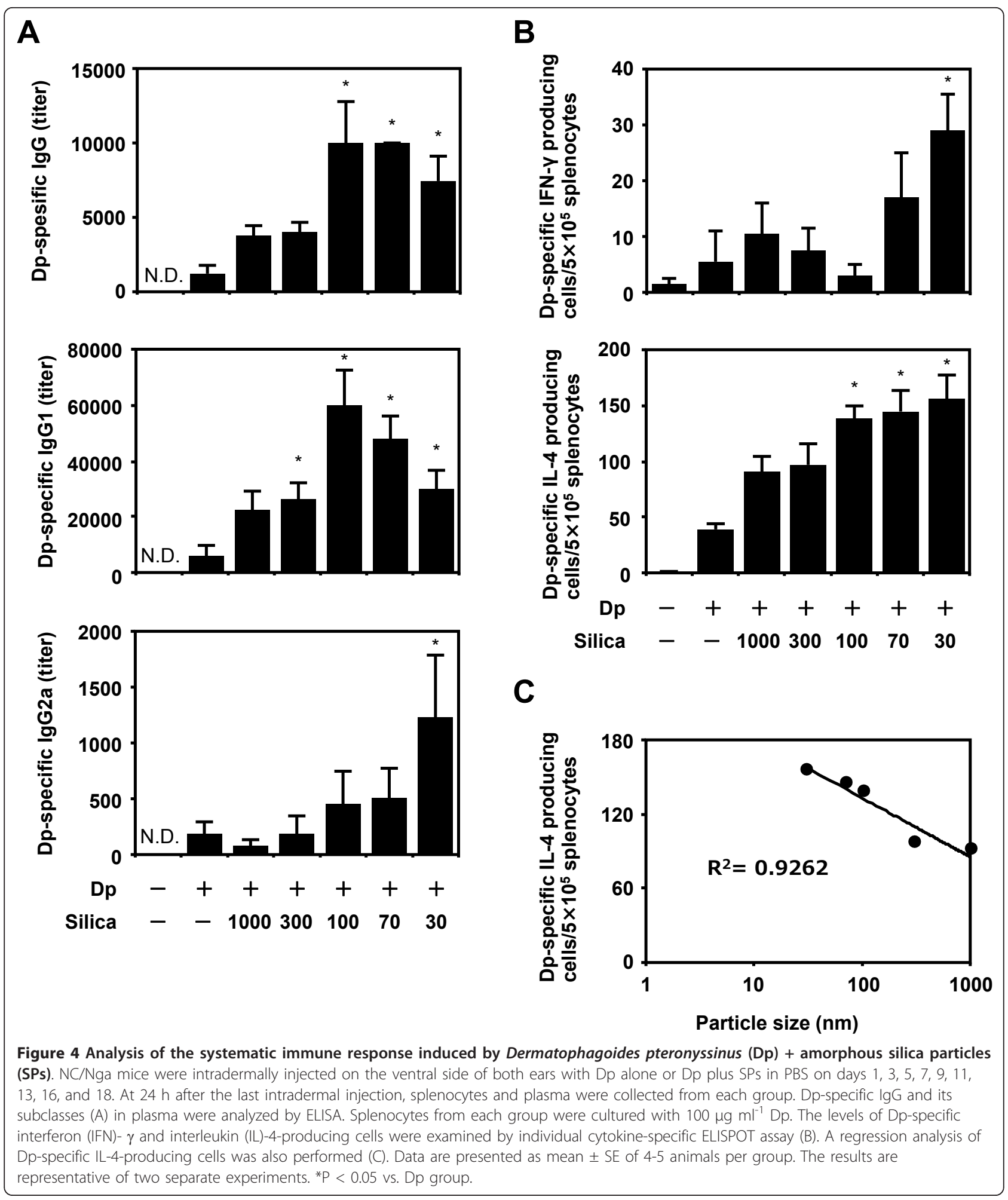

Dp-specific IgE. Thus, the mechanisms different from the mechanisms correlated with Dp-specific IgE production were also involved in this model. Therefore, clarification of the mechanisms of size-dependent aggravation of AD-like skin lesions is important for a risk analysis of nSPs and for designing safer forms of nSPs.

AD skin lesions are characterized by recruitment of lymphocytes, monocytes, eosinophils, and mast cells 
$[29,30]$. These infiltrating cells produce Th2 cytokines including IL-4 and IL-13. These mediators promote Th2 deviation not only in acute eczematous skin lesions but also in systemic adaptive immune systems [31,32]. This Th2 deviation could further compromise barrier function in the skin lesions and complete a potential outside-inside-outside pathogenic loop of AD [33]. Additionally, IL-18 or TSLP plays a critical role in the spontaneous development of AD [34,35]. Considering this, we first performed an analysis of the Th1/Th2 cytokine balance in the skin lesions to clarify the mechanism of AD aggravation by SPs. Although IL-4 levels in the ears increased, IL-5, IL-13, and IFN- $\gamma$ levels decreased following exposure to Dp + SPs compared with those following exposure to Dp alone (Figure 3). There is possible that variously sized SPs induced the expression of Th2 cytokines at an early phase and that this induction might contribute to the aggravation of symptoms. A time-course study is needed to elucidate the correlation between symptoms and inflammatory mediators.

Epithelial cells are major cell source of various proallergic cytokines, such as IL-18 and TSLP. As they are accumulated preferentially in the inflammatory sites of patients with AD, IL-18 and TSLP might be involved in the development of $\mathrm{AD}[16,36]$. To reveal the mechnisms of the nSPs-mediated aggravation of AD-like skin lesion, we performed further analyses to measure IL-18 and TSLP levels in the skin. Interestingly, the IL-18 levels in skin lesions of the Dp + SP groups increased compared to those in the Dp alone group in a SP size-dependent manner (Figure $3 ; \mathrm{R}^{2}=0.91$ ). IL-18 is a cytokine that stimulates mast cells and basophils to release AD-associated molecules, Th2 cytokines, and histamine, without a pathway that involves Ag, IgE, or FcRI. Furthermore, IL-18 induces large amounts of total IgE via natural killer $\mathrm{T}$ and $\mathrm{CD}^{+} \mathrm{T}$ cells [37-39]. These effects of IL-18 finally induce AD-like skin lesions [34]. Considering these, this IL-18 production in a size-dependent manner of SPs show why smaller SPs induced severe AD accompany the production of total IgE. On the other hands, the TSLP levels were only increased following the exposure to Dp + "nSPs" (Figure 3). This result is similar to those of increase in ear thickness, scab and scleredema. TSLP can promote the Th2 cytokine-associated inflammation by directly promoting the effector functions of $\mathrm{CD} 4^{+} \mathrm{Th} 2$ cells, basophils and other granulocyte populations via DC $[35,40]$. Furthermore, it is reported that skin-specific overexpression of TSLP resulted in an AD-like skin lesion $[35,40]$. Thus, these results suggested that TSLP as well as IL-18 might be involved in the development of nSP-mediated AD-like skin lesions in mice. Although we need further mechanistic studies for understanding the nSP-mediated production of IL-18 and TSLP, modification of physicochemical nSPs properties for suppressing IL-18 or TSLP production might be value in the development of safer NMs.

On the other hands, we observed that only treated with nSP70 did not change pathology and local immune responses compared with PBS treated mouse except IL18 productions (our preliminary data). However only IL18 production was tendency to be enhanced by treatment with only silica nanoparticles, the enhancement of IL-18 productions are very slightly (not significant). Thus, we think the evoked inflammation in the ear was mainly attributed to the mixture of nSPs and Dp. Therefore we also must give attention to the interactions of nSPs with antigen to develop of safer NMs.

\section{Conclusions}

In this study, we showed the intradermal injections of SPs and Dp antigen aggravate AD-like skin lesions in size dependent manner. We speculated that particle size affects SPs-induced IL-18 and TSLP inductions enhance the Th2 immune responses related to $\mathrm{Dp}$ and then induce aggravation of $\mathrm{AD}$-like skin lesions. On the other hands, the further studies are required to analyze the mechanisms why IL-18 and TSLP are induced by nSPs. Furthermore, the studies are also required to investigate whether SPs are the risk factor of AD using topical applications of SPs, which is a more likely mode of exposure to NMs. Considering the fact that the nSPs already used in general are smaller than our used ones, our results demand a careful hand. We consider that appropriate regulation of physical and chemical properties of nanoparticles, including sizes mediated by the inductions of IL-18 and TSLP are critical determinants for the design of safer forms of NMs.

\section{Methods}

\section{SPs}

Suspensions of SPs (Micromod Partikeltechnologie GmbH, Rostock, Germany) (25 and $50 \mathrm{mg} \mathrm{ml}^{-1}$ in water) were used; particle size diameters were 1000 , 300, 100, 70, and $30 \mathrm{~nm}$ (designated as mSP1000, nSP300, nSP100, nSP70, and nSP30, respectively). These SPs don't have any coating. SP suspensions were stored at room temperature. The suspensions were sonicated in power of $400 \mathrm{~W}$ for $5 \mathrm{~min}$ at $25^{\circ} \mathrm{C}$ and then vortexed for $1 \mathrm{~min}$ immediately prior to use.

\section{Mice}

Male NC/Nga slc mice were purchased from Nippon SLC (Kyoto, Japan) and used at 8 weeks of age. We used $n=4$ or 5 animals per each assay. All animal experimental procedures were performed in accordance with the institutional ethical guidelines for animal experiments. 


\section{Physicochemical examination of SPs}

SPs were diluted to $0.25 \mathrm{mg} \mathrm{ml}^{-1}$ (nSP30 and nSP70) or $0.5 \mathrm{mg} \mathrm{ml}^{-1}$ (nSP100, nSP300, and mSP1000) with PBS (Sigma-Aldrich, St. Louis, MO, USA), and the average particle size and zeta potential were measured using a Zetasizer Nano-ZS (Malvern Instruments Ltd., Worcestershire, UK) at $25^{\circ} \mathrm{C}$. The mean size and size distribution of SPs were measured using the dynamic light scattering method. The zeta potential was measured by laser Doppler electrophoresis. We perform these measurements using Size and Zeta capillary cell (Malvern Instruments Ltd). The $\mathrm{pH}$ of each particle suspension was measured with an ISFET $\mathrm{pH}$ meter (Shindengen, Tokyo, Japan).

\section{Study protocols}

Mice were divided into seven experimental groups (4 or 5 mice were used in each group): PBS, Dp, Dp + mSP1000, Dp + nSP300, Dp + nSP100, Dp + nSP70, and $\mathrm{Dp}+\mathrm{nSP} 30$ groups. The PBS group received $20 \mu \mathrm{l}$ of PBS. The Dp group received $2.5 \mu \mathrm{g}$ of Dp mite allergen extract (Cosmo Bio LSL, Tokyo, Japan) in $20 \mu \mathrm{l}$ PBS. The Dp + mSP1000, Dp + nSP300, Dp + nSP100, $\mathrm{Dp}+\mathrm{nSP70}$, and $\mathrm{Dp}+\mathrm{nSP} 30$ groups received a combined administration of $2.5 \mu \mathrm{g}$ Dp and $250 \mu \mathrm{g}$ SPs $(1000,300,100,70$, or $30 \mathrm{~nm})$ in $20 \mu \mathrm{l}$ PBS. Mice were intradermally injected on the ventral side of both their ears with Dp alone or Dp plus SPs in PBS on days 1, 3, $5,7,9,11,13,16$, and 18 under anesthesia with pentobarbital (somnopentyl; Kyouritsuseiyaku, Tokyo, Japan). The amount of injected liquid was $10 \mu \mathrm{l}$ per ear. We measured ear thickness on days 0 and 19.

\section{Histological analysis}

At $24 \mathrm{~h}$ after the last intradermal injection, the ears of mice were removed and placed in fixative solution (4\% paraformaldehyde in PBS; Wako, Osaka, Japan). A histopathological examination was performed by the Applied Medical Research Laboratory (AMRL; Osaka, Japan). Several representative symptoms (scab, acanthosis, inflammatory cell infiltration, and scleredema) observed in the $\mathrm{AD}$ of each sample were scored as 0 (none), 1 (very mild), 2 (mild), 3 (moderate), or 4 (severe). Scoring was also performed by AMRL, who was unaware of the treatment assignments. Mast cell numbers in three high-power fields at $\times 400$ magnification were selected randomly and counted.

\section{Blood sampling}

Mice were anesthetized with pentobarbital $24 \mathrm{~h}$ after the last intradermal injection. The chest and abdominal walls were opened, and blood was sampled by cardiac puncture. Then blood was centrifuged at $3000 \mathrm{~g}$ at $4^{\circ} \mathrm{C}$ and we obtained plasma. Plasma was stored at $-80^{\circ} \mathrm{C}$ until assay.

\section{Quantitation of total lgE Abs}

Total IgE in plasma was measured with an ELISA assay kit (BD Biosciences, San Diego, CA, USA) according to the manufacturer's instructions.

\section{Detection of Dp-specific Ab by ELISA}

Antigen-specific Ab levels in plasma were determined by ELISA. ELISA plates (Maxisorp, type 96F; Nunc A/S, Roskilde, Denmark) were coated with $100 \mu \mathrm{g} \mathrm{ml}^{-1} \mathrm{Dp}$ in coating buffer PBS and incubated overnight at $4^{\circ} \mathrm{C}$. The plates were washed with PBS-Tween20, incubated with blocking solution (Block Ace; Dainippon Sumitomo Pharmaceuticals, Osaka, Japan) at room temperature (RT) for $2 \mathrm{~h}$, and plasma dilutions were added to the antigen-coated plates. After a 2-h RT incubation, the coated plates were washed with PBS-Tween 20 and incubated with a HRP-conjugated goat anti-mouse IgG or IgG1 solution or a biotin-conjugated goat anti-mouse IgG2a or IgE detection Ab solution (Southern Biotechnology Associates, Birmingham, AL, USA) at RT for 2 h. To detect IgG2a or IgE, the plates were washed with PBS-Tween 20 and then incubated with HRP-coupled streptavidin (Southern Biotechnology Associates) for 30 $\mathrm{h}$ at RT. After the incubation, the color reaction was developed with tetra methyl benzidine (MOSS, Inc.; Pasadena, MD, USA), stopped with $2 \mathrm{~N} \mathrm{H}_{2} \mathrm{SO}_{4}$, and measured at $\mathrm{OD}_{450-620}$ on a microplate reader.

\section{Isolation of splenocytes}

Spleens were aseptically removed and placed in RPMI 1640 (Wako) supplemented with $10 \%$ FBS, $10 \mathrm{ml} \mathrm{L}^{-1}$ of a $100 \times$ nonessential amino acid solution (Gibco, Invitrogen, Carlsbad, CA, USA), $50 \mu \mathrm{M}$ 2-mercaptoethanol (Gibco), and 1\% antibiotic cocktail (Pennicilin; $10000 \mathrm{U} /$ $\mathrm{mL}$, Streptomycin; $10000 \mu \mathrm{g} / \mathrm{mL}$, amphotericin B; 25 $\mu \mathrm{g} / \mathrm{mL}$ ) (Gibco). A single-cell suspension of splenocytes was treated with ammonium chloride to lyse the red blood cells, which were washed, counted, and suspended in RPMI 1640 to a final concentration of $1 \times 10^{7}$ cells $\mathrm{ml}^{-1}$.

\section{ELISPOT assay}

An ELISPOT assay was performed to detect interferon (IFN) $-\gamma$ and IL-4 producing cells. Dp-specific cytokine responses were evaluated by culturing the splenocytes $(5$ $\times 10^{5}$ cells well $\left.{ }^{-1}\right)$ stimulated with $\mathrm{Dp}\left(100 \mu \mathrm{g} \mathrm{ml}^{-1}\right)$ in vitro. After a 24 -h incubation at $37^{\circ} \mathrm{C}$, the plate was washed, and the IFN- $\gamma$ - and IL-4-producing cells were measured with an ELISPOT assay kit (BD Biosciences) according to the manufacturer's instructions. 


\section{Quantitation of cytokine protein levels in ear tissue supernatants}

At $24 \mathrm{~h}$ after the last intradermal injection, mice ears were removed and stored at $-80^{\circ} \mathrm{C}$. Later, the ears were homogenized with cell extraction buffer (Invitrogen). The homogenates were then centrifuged at 10,000 rpm for $5 \mathrm{~min}$, and the supernatants were stored at $-30^{\circ} \mathrm{C}$. ELISAs for IFN- $\gamma$ (BD Biosciences), IL-4, IL-5, IL-13 (eBioscience, San Diego, CA, USA), IL-18 (MBL, Nagoya, Japan), and thymic stromal lymphopoietin (TSLP; R\&D Systems, Minneapolis, MN, USA) in the ear tissue supernatants were performed according to the manufacturer's instructions.

\section{Statistical analysis}

All data are presented as mean $\pm \mathrm{SE}$. The significant differences among groups were determined by ANOVA. Differences between the experimental groups and the control group were determined by Williams' test. P < 0.05 was considered significant.

\section{Acknowledgements}

This study was supported, in part, by Grants-in-Aid for Scientific Research from the Ministry of Education, Culture, Sports, Science and Technology of Japan (MEXT) and from the Japan Society for the Promotion of Science (JSPS); and by the Knowledge Cluster Initiative (MEXT); by Health Labour Sciences Research Grants from the Ministry of Health, Labour and Welfare of Japan (MHLW); by a Global Environment Research Fund from the Ministry of the Environment; by Food Safety Commission (Cabinet Office); by The Cosmetology Research Foundation; by The Smoking Research Foundation; and by The Takeda Science Foundation.

\section{Author details \\ ${ }^{1}$ Laboratory of Toxicology and Safety Science, Graduate School of Pharmaceutical Sciences, Osaka University, 1-6, Yamadaoka, Suita, Osaka 565- 0871, Japan. ${ }^{2}$ Laboratory of Biopharmaceutical Research (Pharmaceutical Proteomics), National Institute of Biomedical Innovation, 7-6-8, Saito-Asagi, Ibaraki, Osaka 567-0085, Japan. ${ }^{3}$ The Center for Advanced Medical Engineering and Informatics, Osaka University, 1-6, Yamadaoka, Suita, Osaka 565-0871, Japan.}

\section{Authors' contributions}

TH and TY designed the study; TH, HN, TY, S. Tochigi, Kl, MU and TA performed experiments; TH and TY collected and analyzed data; TH and TY wrote the manuscript; KN, YA, YY, HK, NI and S. Tsunoda gave technical support and conceptual advice. YT supervised the all of projects. All authors discussed the results and commented on the manuscript. All authors have read and approved the final manuscript.

\section{Competing interests}

The authors declare that they have no competing interests.

Received: 21 September 2011 Accepted: 2 February 2012 Published: 2 February 2012

\section{References}

1. Bowman DM, van Calster G, Friedrichs S: Nanomaterials and regulation of cosmetics. Nat Nanotechnol 2010, 5:92.

2. Liu D, Gu N: Nanomaterials for fresh-keeping and sterilization in food preservation. Recent Pat Food Nutr Agric 2009, 1:149-154.

3. Salata O: Applications of nanoparticles in biology and medicine. $J$ Nanobiotechnology 2004, 2:3.
4. Choksi AN, Poonawalla T, Wilkerson MG: Nanoparticles: a closer look at their dermal effects. J Drugs Dermatol 2010, 9:475-481.

5. Nel A, Xia T, Madler L, Li N: Toxic potential of materials at the nanolevel. Science 2006, 311:622-627.

6. Shimizu M, Tainaka H, Oba T, Mizuo K, Umezawa M, Takeda K: Maternal exposure to nanoparticulate titanium dioxide during the prenatal period alters gene expression related to brain development in the mouse. Part Fibre Toxicol 2009, 6:20.

7. Nishimori H, Kondoh M, Isoda K, Tsunoda S, Tsutsumi Y, Yagi K: Silica nanoparticles as hepatotoxicants. Eur J Pharm Biopharm 2009, 72:496-501.

8. Yamashita K, Yoshioka Y, Higashisaka K, Mimura K, Morishita Y, Nozaki M, Yoshida T, Ogura T, Nabeshi H, Nagano K, et al: Silica and titanium dioxide nanoparticles cause pregnancy complications in mice. Nat Nanotechnol 2011, 6:321-328.

9. Knopp D, Tang D, Niessner R: Review: bioanalytical applications of biomolecule-functionalized nanometer-sized doped silica particles. Anal Chim Acta 2009, 647:14-30.

10. Martin KR: The chemistry of silica and its potential health benefits. J Nutr Health Aging 2007, 11:94-97.

11. Nabeshi H, Yoshikawa T, Matsuyama K, Nakazato Y, Matsuo K, Arimori A, Isobe M, Tochigi S, Kondoh S, Hirai T, et al: Systemic distribution, nuclear entry and cytotoxicity of amorphous nanosilica following topical application. Biomaterials 2011, 32:2713-2724.

12. Nabeshi H, Yoshikawa T, Matsuyama K, Nakazato Y, Arimori A, Isobe M, Tochigi S, Kondoh S, Hirai T, Akase T, et al: Size-dependent cytotoxic effects of amorphous silica nanoparticles on Langerhans cells. Pharmazie 2010, 65:199-201.

13. Bohle B, Schwihla H, Hu HZ, Friedl-Hajek R, Sowka S, Ferreira F, Breiteneder H, Bruijnzeel-Koomen CA, de Weger RA, Mudde GC, et al: Longlived Th2 clones specific for seasonal and perennial allergens can be detected in blood and skin by their TCR-hypervariable regions. J Immunol 1998, 160:2022-2027.

14. Flohr C, Johansson SG, Wahlgren CF, Williams H: How atopic is atopic dermatitis? J Allergy Clin Immunol 2004, 114:150-158.

15. Schafer T, Heinrich J, Wjst M, Adam H, Ring J, Wichmann HE: Association between severity of atopic eczema and degree of sensitization to aeroallergens in schoolchildren. J Allergy Clin Immunol 1999, 104:1280-1284.

16. Bieber T: Atopic dermatitis. Ann Dermatol 2010, 22:125-137.

17. Plotz SG, Ring J: What's new in atopic eczema? Expert Opin Emerg Drugs 2010, 15:249-267.

18. Annesi-Maesano I, Caillaud D, Lavaud F, Moreau D, Le Moullec Y, Taytard A, Pauli G, Charpin D: Exposure to fine air particles and occurrence of allergic diseases: results of ISAAC-France phase 2. Arch Pediatr 2009, 16:299-305.

19. Annesi-Maesano I, Moreau D, Caillaud D, Lavaud F, Le Moullec Y, Taytard A, Pauli G, Charpin D: Residential proximity fine particles related to allergic sensitisation and asthma in primary school children. Respir Med 2007, 101:1721-1729.

20. Yanagisawa R, Takano H, Inoue K, Koike E, Kamachi T, Sadakane K, Ichinose $T$ : Titanium dioxide nanoparticles aggravate atopic dermatitislike skin lesions in NC/Nga mice. Exp Biol Med (Maywood) 2009, 234:314-322.

21. Yanagisawa R, Takano H, Inoue KI, Koike E, Sadakane K, Ichinose T: Size effects of polystyrene nanoparticles on atopic dermatitislike skin lesions in NC/NGA mice. Int I Immunopathol Pharmacol 2010, 23:131-141.

22. Kawakami T, Ando T, Kimura M, Wilson BS, Kawakami Y: Mast cells in atopic dermatitis. Curr Opin Immunol 2009, 21:666-678.

23. Yamashita H, Michibata Y, Mizukami H, Ogihara Y, Morita A, Nose M: Dermal mast cells play a central role in the incidence of scratching behavior in mice induced by multiple application of the hapten, 2,4,6trinitrochlorobenzene. Exp Dermatol 2005, 14:438-444.

24. Stone KD, Prussin C, Metcalfe DD: IgE, mast cells, basophils, and eosinophils. J Allergy Clin Immunol 2010, 125:S73-80.

25. Hirai T, Yoshikawa T, Nabeshi H, Yoshida T, Tochigi S, Uji M, Ichihashi K, Akase T, Yamashita T, Yamashita K, Nagano K, Abe Y, Kamada H, Tsunoda S, Yoshioka Y, Itoh N, Tsutsumi Y: Size-dependent immune-modulating effect of amorphous nanosilica particles. Pharmazie

26. Tran KK, Shen $\mathrm{H}$ : The role of phagosomal pH on the size-dependent efficiency of cross-presentation by dendritic cells. Biomaterials 2009, 30:1356-1362. 
27. Aral M, Arican O, Gul M, Sasmaz S, Kocturk SA, Kastal U, Ekerbicer HC: The relationship between serum levels of total IgE, IL-18, IL-12, IFN-gamma and disease severity in children with atopic dermatitis. Mediators Inflamm 2006, 2006:73098.

28. Hon KL, Lam MC, Leung TF, Wong KY, Chow CM, Fok TF, Ng PC: Are agespecific high serum IgE levels associated with worse symptomatology in children with atopic dermatitis? Int J Dermatol 2007, 46:1258-1262.

29. Kay AB, Ying S, Varney V, Gaga M, Durham SR, Mogbel R, Wardlaw AJ, Hamid Q: Messenger RNA expression of the cytokine gene cluster, interleukin 3 (IL-3), IL-4, IL-5, and granulocyte/macrophage colonystimulating factor, in allergen-induced late-phase cutaneous reactions in atopic subjects. J Exp Med 1991, 173:775-778.

30. Rousset F, Robert J, Andary M, Bonnin JP, Souillet G, Chretien I, Briere F, Pene J, de Vries JE: Shifts in interleukin-4 and interferon-gamma production by $T$ cells of patients with elevated serum IgE levels and the modulatory effects of these lymphokines on spontaneous $\lg E$ synthesis. J Allergy Clin Immunol 1991, 87:58-69.

31. Leung DY, Boguniewicz M, Howell MD, Nomura I, Hamid QA: New insights into atopic dermatitis. J Clin Invest 2004, 113:651-657.

32. Liu FT, Goodarzi H, Chen HY: IgE, Mast Cells, and Eosinophils in Atopic Dermatitis. Clin Rev Allergy Immunol 2011.

33. Elias PM, Steinhoff M: "Outside-to-inside" (and now back to "outside") pathogenic mechanisms in atopic dermatitis. J Invest Dermatol 2008 128:1067-1070.

34. Konishi H, Tsutsui H, Murakami T, Yumikura-Futatsugi S, Yamanaka K, Tanaka M, Iwakura Y, Suzuki N, Takeda K, Akira S, et al: IL-18 contributes to the spontaneous development of atopic dermatitis-like inflammatory skin lesion independently of lgE/stat6 under specific pathogen-free conditions. Proc Natl Acad Sci USA 2002, 99:11340-11345.

35. Ziegler SF, Artis D: Sensing the outside world: TSLP regulates barrier immunity. Nat Immunol 2010, 11:289-293.

36. Terada M, Tsutsui H, Imai Y, Yasuda K, Mizutani H, Yamanishi K, Kubo M, Matsui K, Sano H, Nakanishi K: Contribution of IL-18 to atopic-dermatitislike skin inflammation induced by Staphylococcus aureus product in mice. Proc Natl Acad Sci USA 2006, 103:8816-8821.

37. Yoshimoto $T$, Min B, Sugimoto T, Hayashi N, Ishikawa $Y$, Sasaki $Y$, Hata H, Takeda K, Okumura K, Van Kaer L, et al: Nonredundant roles for CD1drestricted natural killer $T$ cells and conventional CD4+ T cells in the induction of immunoglobulin $\mathrm{E}$ antibodies in response to interleukin 18 treatment of mice. J Exp Med 2003, 197:997-1005.

38. Yoshimoto T, Nakanishi K: Roles of IL-18 in basophils and mast cells. Allergol Int 2006, 55:105-113.

39. Yoshimoto T, Tsutsui H, Tominaga K, Hoshino K, Okamura H, Akira S, Paul WE, Nakanishi K: IL-18, although antiallergic when administered with IL-12, stimulates IL-4 and histamine release by basophils. Proc Natl Acad Sci USA 1999, 96:13962-13966.

40. Fang C, Siew LQ, Corrigan CJ, Ying S: The role of thymic stromal lymphopoietin in allergic inflammation and chronic obstructive pulmonary disease. Arch Immunol Ther Exp (Warsz) 2010, 58:81-90.

doi:10.1186/1743-8977-9-3

Cite this article as: Hirai et al:: Amorphous silica nanoparticles sizedependently aggravate atopic dermatitis-like skin lesions following an intradermal injection. Particle and Fibre Toxicology 2012 9:3.

\section{Submit your next manuscript to BioMed Central and take full advantage of:}

- Convenient online submission

- Thorough peer review

- No space constraints or color figure charges

- Immediate publication on acceptance

- Inclusion in PubMed, CAS, Scopus and Google Scholar

- Research which is freely available for redistribution 